\title{
CRISIS INTERVENTIONS IN ACUTE EMERGENCY SITUATIONS
}

\section{KRISENINTERVENTION IM RAHMEN VON AKUTEN BELASTUNGSSITUATIONEN}

\author{
Monika Czamler \\ Geschäftsfeldleitung der Krisenhilfe OÖ, Linz, Austria
}

\begin{abstract}
SUMMARY
In the article about crisis intervention after incriminating events, I start by explaining the specific terms, since in recent years different terms have been used, which have repeatedly caused uncertainty.

How might people react to extreme stress and what are factors of protection and risk?

Further I will explain the effect of psychosocial support as well as the structure and forms of interventions. The basic principles of interventions and the 10 essential intervention steps are described. In order to be able to provide targeted assistance, it is necessary for the psychosocial emergency aid staff to know about the experience of those affected in acute emergency situations.

In order to ensure that psychosocial emergency helpers are able to recover from their missions or taking care of those affected, it is necessary to know the risks and take them into account while planning the auxiliary structure.
\end{abstract}

Key words: emergency - trauma - crisis - Protection and risk factors - Effective factors of psychosocial support Structure of psychosocial acute intervention - forms of intervention - Experience in acute emergency situations Basic principles of interventions - 10 Intervention steps in emergencies - Dangers to psychosocial emergency helpers

\section{ZUSAMMENFASSUNG}

Im Beitrag über die Krisenintervention nach belastenden Ereignissen gehe ich am Anfang auf die Definitionen der Begriffe ein, da in den letzten Jahren unterschiedliche Terminologien verwendet werden, die immer wieder für Unklarheiten sorgen.

Wie können Menschen auf Extremstress reagieren und welche Schutz- und Risikofaktoren gibt es?

Weiter gehe ich auf Wirkfaktoren in der psychosozialen Unterstützung ein, sowie auf Struktur und Formen der Interventionen. Dabei werden die Grundprinzipien der Interventionen und zusammengefasst die 10 wesentlichen Interventionsschritte beschrieben. Um gezielt hilfreiche Interventionen setzen zu können ist es für die psychosozialen Notfallhelfer notwendig, über das Erleben der Betroffenen in akuten Notfallsituationen Bescheid zu wissen.

Damit psychosoziale Notfallhelfer auch selbst bei einem solchen Einsatz bzw. der Betreuung Betroffener keinen gesundheitlichen Schaden nehmen, ist es notwendig die Gefahren zu kennen und in der Planung der Hilfsstruktur darauf Rücksicht zu nehmen.

Schlüsselwörter: Notfall - Trauma - Krise - Schutz- und Risikofaktoren - Wirkfaktoren psychosozialer Unterstützung Struktur der psychosozialen Akutintervention - Interventionsformen - Das Erleben in akuten Notfallsituationen Grundprinzipien der Interventionen - 10 Interventionsschritte in Notfällen - Gefahren für psychosoziale Notfallhelferinnen

\section{Einleitung}

Es gab in den letzten Jahren eine rasante Entwicklung im Bereich der Krisenintervention - vor allem die Krisenintervention nach belastenden Ereignissen rückte in den Fokus der Aufmerksamkeit. Die ambulante Krisenintervention bei Lebensveränderungskrisen und psychiatrischen Notfällen sind in meinem Beitrag nicht erwähnt, wenn ich hier den Begriff Krisenintervention verwende, ist damit die Unterstützung von Personen nach traumatischen Ereignissen durch multiprofessionell zusammengesetzte Teams gemeint, wobei es natürlich immer Überschneidungen gibt.

Notfallinterventionen sind Maßnahmen die sich auf das Erleben und Verhalten während und nach Notfällen beziehen, egal zu welchem Zeitpunkt. Sie reichen von den ersten Minuten und Stunden nach dem Ereignis, über die Zeit der psychischen Stabilisierung, wo es darum geht wieder „Boden unter den Füßen“ zu bekommen bis zur individuellen Nachbetreuung.

Großereignisse wie das Zugunglück in Eschede (Deutschland), das Unglück in Kaprun oder auch die Hochwasserkatastrophe 2002 haben deutlich gemacht, dass es psychosoziale Betreuungssysteme in/nach Katastrophen braucht. In der weiteren Entwicklung haben wir uns auch in OÖ Gedanken gemacht wie die Unterstützung von Betroffenen nach Einzelereignissen organisiert werden kann.

Die Begriffe „Notfall“, „Trauma“, „Krise“ werden in diesem Bereich sehr uneinheitlich verwendet und bleiben deswegen oft unscharf, daher folgende Definitionen:

\section{Notfall}

„Notfälle sind Ereignisse, die aufgrund ihrer subjektiv erlebten Intensität physisch und/oder 
psychisch, als so beeinträchtigend erlebt werden, dass sie zu negativen Folgen in der physischen und/oder psychischen Gesundheit führen können.“ (Lasogga und Gasch 2004)

Wesentlich an dieser Definition ist, dass nur subjektive Kriterien herangezogen werden, d. h. ein und dasselbe Ereignis kann von den unterschiedlichen Menschen sehr unterschiedlich erlebt und bewertet werden und damit auch zu unterschiedlichen Reaktionen führen.

\section{Trauma}

Ein psychisches Trauma ist ein plötzlich auftretendes Ereignis, das auf den Betroffenen sehr bedrohlich wirkt und nicht bewältigbar erscheint. Es verletzt die Grundannahmen über die Welt und die eigene Person. Ein Trauma löst intensive Gefühle (Entsetzen, Wut, Verzweiflung, Angst) aus und führt zu einem Zustand anhaltender emotionaler Betäubung. Es kann langfristige psychische Symptome und Störungen verursachen.

Nach dieser Definition werden sowohl das Ereignis, dessen Bewertung und zusätzlich der möglichen negativen Folgen als Kriterien herangezogen. Ein Trauma ist dann ein ,vitales Diskrepanzerlebnis zwischen bedrohlichen Situationsfaktoren und den individuellen Bewältigungsmöglichkeiten, das so eine dauerhafte Erschütterung des Selbst- und Weltverständnisses bewirkt" (Fischer und Riedesser 2003)

\section{Krise}

„Unter Krise versteht man den Verlust des seelischen Gleichgewichts infolge akuter Überforderung eines gewohnten Verhaltens-/ Bewältigungssystems durch belastende äußere oder innere Ereignisse“ (Riecher-Rössler 2004).

Der Begriff „Krise“ wird sehr unterschiedlich verwendet, es gibt auch die Unterscheidung zwischen traumatische Krise und Lebensveränderungskrise. Krise wird meist für die Lebensveränderungskrise verwendet und für die „traumatische Krise“ der Begriff Notfall und damit ist auch die Differenzierung klarer.

Welche kurz- und langfristigen Folgen ein Trauma hat, hängt von verschiedenen Schutz- und Risikofaktoren ab. Sie wurden bisher vor allem in Bezug auf die Entwicklung einer posttraumatischen Belastungsstörung (PTBS) untersucht, der häufigsten Folgestörungen nach einem Notfall.

\section{Als Schutzfaktoren sind zu erwähnen}

- Eine dauerhaft gute Beziehung zu mindestens einer primären Bezugsperson;

- Verlässliche Unterstützung durch Bezugspersonen (Ehe- Lebenspartner);

- Aufwachsen in einer Großfamilie;

- Sicheres Bindungsverhalten;

- Robustes, aktives, kontaktfreudiges Temperament;

- Geringe psychosoziale Gesamtbelastung.
Das gute soziale Netz, ist eine der Variablen die übereinstimmend in unterschiedlichen Untersuchungen als ,vor negativen Folgen des Notfalls schützend“ beurteilt werden.

Es gibt verschiedene Faktoren die die Verarbeitung des Traumas erschweren. Es wird unterschieden zwischen Faktoren die das Ereignis, die Person und die Reaktionen während und nach dem Notfall betreffen. Nicht zuletzt spielt natürlich auch die Reaktion der Umwelt eine große Rolle, ob ein Ereignis gut bewältigt werden kann oder nicht.

\section{Folgende Ereignisfaktoren gelten als Risikofaktoren}

- Das Ereignis ist von sehr starker Intensität und dauert sehr lange, z. B. es geschieht ein schweres Zugunglück und die Bergung der Verletzten dauert einige Stunden.

- Wenn sich das potentiell traumatisierende Ereignis wiederholt - wie z.B. sexuelle Übergriffe.

- Durch das Ereignis erleidet der/die Betroffene erhebliche Verletzungen bzw. die Betroffenen erleben es als Bedrohung des Lebens. Das kann auch bei einem Überfall die Bedrohung mit einer Waffe sein.

- Wenn betroffene Menschen keine bzw. sehr wenig eigene Kontrolle über das Geschehen haben.

- Ein wesentlicher Faktor der die Bewältigung erschwert ist, wenn es sich um eine absichtliche Schädigung bzw. Verletzung handelt und das Ereignis ein von Menschen zu verantwortendes ist.

\section{Die persönlichen Risikofaktoren}

- Allgemeiner Lebensstress.

- Belastungen in der Kindheit, wie z.B. Misshandlungen, früher Verlust eines Elternteils

- Frühere Traumata.

- Psychische Erkrankungen in der Familie.

- Niedriger sozioökonomischer Status.

Weitere Faktoren die das PTBS Risiko (nach Steil, 2001)

- Das Ausmaß der unwillkürlichen Dissoziation während des Traumas.

- Akute Symptomatik (je stärker, desto höher das PTBS Risiko).

- Ausmaß dysfunktionaler Kognitionen., d.h. problematischer Gedanken.

- Ausmaß der Vermeidung traumabezogener Reize.

- Bleibend körperliche Beeinträchtigung/Behinderung

Die Reaktionen der Angehörigen, Freunden, Kollegen, aber auch von Behördenvertretern, Journalisten etc. können die mittel- und langfristige Bewältigung eines Traumas wesentliche beeinflussen.

Wenn fremde Hilfe ausbleibt (praktisch, finanziell oder rechtlich), es wenig oder keine soziale Unterstützung gibt oder Vorwürfe bzw. Schuldzuweisungen, 
ist dies für die Betroffenen eine zusätzliche Belastung. Betroffene schildern in Betreuungssettings öfter, dass sie von ihrem persönlichen Umfeld immer wieder angeregt werden zu erzählen, was passiert ist, nach Details fragen und dadurch ein ständiges Erinnern an der Geschehene aktiviert wird - Triggerung.

Bei plötzlichen Notfällen reagiert ein Mensch zunächst ,biologisch“ d. h. er greift auf automatische, angeborene, ,archaische“ Verhaltensprogramme zurück. Ein typischer Ablauf wäre demnach nach Lasogga Gasch:

Erschrecken - Regungslosigkeit - Erstarrung Innehalten der Atmung - Verstummen - Konzentration auf ,stabile Kernzonen des autonomen Selbst“;

Dann erfolgt eine Orientierungsreaktion: Herzfrequenzsteigerung - Ausrichten des Kopfes - Anspannung des Körpers - Aufmerksamkeitssteigerung Furcht - Verhaltensanpassung durch Flucht oder Kampf. In einer weiteren Phase werden dann individuell erworbene „psychologische“ Mechanismen aktiv, die als Coping-Mechanismen bezeichnet werden können.

\section{Wie kann psychosoziale Unterstützung in dieser Situation wirken?}

Psychosoziale Unterstützung in der Akutbetreuung/ Krisenintervention wird definiert als die Summe von psychologischen wie auch sozialen Unterstützungsmaßnahmen für Betroffene in der Akutsituation. Diese Maßnahmen zielen auf die Bedürfnisse von Individuen oder Gruppen ab, fokussierend auf Resilienz der Individuen, Gruppen und Gemeinschaften (Reference Centre of Psychosocial Support 2012).

\section{Wirkfaktoren psychosozialer Unterstützung:}

Hobfoll et al. (2007) zeigten in ihrer Arbeit fünf zentrale Elemente auf, welche sofern sie in Interventionen nach Notfällen enthalten sind, nachgewiesenermaßen positive Wirkung entfalten.

\section{Sicherheit}

Betrifft einerseits das eigene Gefühl von Sicherheit, aber auch die objektiven Aspekte der Sicherheit wie z. B. Bereitstellen eines sicheren Ortes. Das beinhaltet auch Schutz vor Überflutung bezüglich Informationen und vor den Medien. Gleichzeitig ist aber auch gezielte Informationsweitergabe über Verbleib und Zustand von Angehörigen, Freunden etc. sehr notwendig.

\section{Verbundenheit}

Verbundenheit ist ein bedeutsamer Wirkfaktor und ein zentrales Element für Erholung. Um Verbundenheit $\mathrm{zu}$ fördern wird einerseits soziale Unterstützung begünstigt und andererseits werden auch soziale Aktivitäten gefördert. In der Akutintervention geht es vor allem um Zusammenführung von Familien und Freunden und die Nutzung von sozialen Netzwerken der Betroffenen.

\section{Stressreduktion}

Stressreduktion und Distanzierung wird vor allem durch die Förderung des Gefühls von Ruhe gefördert. Da es nach dem Erleben eines potentiell traumatisierenden Ereignisses kann es zu einer Übererregtheit, erhöhter Ängstlichkeit und emotionaler Labilität der Betroffenen kommen. Dem soll durch Stressreduktion und Distanzierung und Maßnahmen der Psychoedukation entgegengewirkt werden.

\section{Selbst- und kollektive Wirksamkeit}

Selbst- und kollektive Wirksamkeit bedeuten die Bereitstellung von Möglichkeiten der Mitwirkung, die Förderung eigener Handlungs- und Entscheidungsmöglichkeiten. Zentrale Elemente dafür sind die Bereitstellung von Ressourcen, geeignete und gute Strukturen. In der Akutintervention bedeutet dass die Vorgabe eines klaren Rahmens in dem Entscheidungs- und Handlungsmöglichkeit gegeben sind.

\section{Hoffnung}

Hoffnung unterstützt Erholung ebenfalls, es geht darum Hoffnung zu vermitteln, aber auch Ermutigungen auszusprechen. In der Akutintervention geht es oft darum kleine Schritte in die Zukunft zu wagen, in dem schwierige Schritte gemeinsam besprochen werden und somit bewältigbar werden.

\section{Struktur der psychosozialen Akutintervention}

Wir unterscheiden in der Organisation zwischen Einzelnotfällen, Großschadensereignissen und Katastrophen.

Bei Einzelnotfällen geht es um ein Ereignis wie Autounfall, Überfall, Suizid etc. Es können auch mehrere Menschen betroffen sein. Es handelt sich meist um den Familienverband, Freunde und ev. Kollegen. In dieser Situation sind zwei KollegInnen im Einsatz vor Ort.

Großschadensereignisse erfordern eine komplexere Organisation, es sind mehrere KollegInnen im Einsatz und zusätzliche Konzepte sind erforderlich, wie Einsatzleitung und Sicherstellung des Informationsflusses zwischen der Einsatzleitung, den KollegInnen und anderen Organisationen.

Katastrophe ist ein großes Ereignis, dies kann eine technische oder Naturkatastrophe bzw. ein von Menschenhand verursachtes Ereignis sein. Die Katastrophe kann nur vom Landeshauptmann benannt werden und auch in der psychosozialen Unterstützung der Betroffenen laufen die geplanten Maßnahmen an.

\section{Wir unterscheiden die betroffenen \\ Personengruppen und zwar}

- Direkte Notfallopfer oder Primäropfer, diese haben selbst einen Notfall erlitten und das Ausmaß der psychischen Belastung kann sehr unterschiedlich sein.

- Indirekte Notfallopfer oder Sekundäropfer werden indirekt mit dem Notfall konfrontiert wie z.B. Augenzeugen, Angehörige, Verursacher, Medienvertreter und Helfer. 


\section{Interventionsformen}

In der Praxis ist die Krisenintervention nach Notfällen einerseits spezifischer als die allgemeine Krisenintervention, da sie sich nur mit den psychischen Folgen von potentiell traumatisierenden Ereignissen beschäftigt. Andererseits geht sie zeitlich über die Akutbetreuung hinaus da sie auch Langzeitfolgen berücksichtigt.

Wir unterscheiden in 4 verschiedene Phasen in der Akutbetreuung:

\section{Akuthilfe}

Das vorrangige Ziel der Akuthilfe ist, eine psychische Dekompensation der Betroffenen zu verhindern bzw. aufzufangen. Die Dauer der Akuthilfe beträgt zumeist einige Stunden, bei Großschadensereignissen bzw. Katastrophen auch einige Tage. Die Akuthilfe findet meist vor Ort statt. Die Interventionen haben den Charakter einer akuten Krisenintervention.

\section{Psychische Stabilisierung}

Psychische Stabilisierung umfasst die Unterstützung von Verletzten und Angehörigen, nachdem sie vom Ort des Geschehens weggebracht worden sind. Sie sind entweder im Krankenhaus oder Zuhause. Die Unterstützung wird bei Bedarf aber auch Zeugen, Nachbarn, Anrainern in den Tagen und Wochen nach dem Ereignis angeboten. Auch Einsatzkräfte nach besonders belastenden Einsätzen werden in dieser Phase unterstützt. Die Interventionen haben einerseits präventiven aber auch behandelnden Charakter. Sie dienen der Behandlung manifester Symptome und sollen die Entstehung von Folgestörungen verhindern.

\section{Individuelle Weiterbetreuung und - behandlung}

Individuelle Weiterbetreuung und - behandlung für besonders betroffene oder traumatisierte Personen setzt ein, wenn aufgetretene Symptome nicht vergehen oder sich zu psychischen Folgestörungen verfestigen. Die Behandlung erfolgt meist in Form einer speziellen Traumatherapie.

\section{Information und Schulung}

Information und Schulung ist vor allem für Einsatzkräfte und psychosoziale NotfallhelferInnen von Bedeutung, die immer wieder mit Notfällen konfrontiert sind. Diese können durch Schulung auf künftige Einsätze und die damit verbundenen psychischen Belastungen vorbereitet werden um dadurch im Ernstfall besser damit umgehen zu können.

\section{Das Erleben in akuten Notfallsituationen}

Notfälle sind Ausnahmesituationen. Der übliche Ablauf der Dinge ist unterbrochen. Zentrale Bereiche des Alltags sind außer Kraft gesetzt. Das Erleben und Verhalten ändert sich zum Teil radikal.

\section{Wahrnehmungen}

Betroffene sind in Notfällen extremen Eindrücken ausgesetzt. Der Anblick von Zerstörung, Blut und
Verletzungen, das Hören von Schreien, Weinen sind für viele Menschen Angst auslösend. Auch das Empfinden ist verändert, manche Menschen spüren in Notfällen fast nichts, haben kein Schmerzempfinden, andere haben eine sehr intensive Wahrnehmung - beides ist sehr belastend.

\section{Zeiterleben}

Aufgrund der extremen Wachheit, in der die ganze Aufmerksamkeit auf die ablaufenden Ereignisse fokussiert ist, wird die Zeitwahrnehmung gedehnt. Sekunden können ewig dauern. Wenn die Erregung andauert bleibt auch das Zeiterleben verändert. Vergangenheit und Zukunft scheinen abgeschnitten.

\section{Handlungen - Pläne}

Geplante Handlungen werden plötzlich unterbrochen und können erst einmal nicht wieder aufgenommen werden. Das kann einen Bruch bewirken, dass ,nichts mehr ist, wie es war". Die Betroffenen fühlen sich oft orientierungslos.

\section{Kontrollierbarkeit}

In Notfällen ist der übliche Ablauf der Dinge auf gefährliche Weise unterbrochen und können nicht mehr beeinflusst werden. Auch die eigenen Reaktionen geraten außer Kontrolle. Kontrollverlust ist für die meisten Menschen sehr unangenehm.

\section{Selbstbild}

Die veränderten Wahrnehmungen, mögliche Schmerzen durch Verletzungen, der Verlust der Eigenständigkeit sowie die Ungewissheit über das Ausmaß der Folgen, können das Selbstbild erschüttern. Es entsteht das Gefühl den Boden unter den Füßen zu verlieren.

\section{Soziale Ordnung}

Notfälle können die soziale Ordnung außer Kraft setzen, bisher selbständige Menschen sind plötzlich auf Hilfe angewiesen. Betroffene haben oft keinen Einfluss auf wichtige, sie betreffende Entscheidungen. Die erlebt Hilflosigkeit wird oft als sehr unangenehm und beschämend erlebt.

\section{Grundprinzipien der Interventionen}

Ruhiges und sicheres Auftreten der Notfallhelfer gibt Sicherheit und nimmt bei den Betroffenen etwas von ihrer Angst.

Es ist sehr wesentlich, sich in der Betreuung nicht in den allgemeinen vorhandenen Stress hineinziehen zu lassen. Verlangsamung bei den Notfallhelfern hilft den Betroffenen gegen die Übererregung.

Während und nach Notfällen ist das Chaos groß, auch das innere Chaos der Betroffenen. Notfallhilfe braucht Struktur, die klare Vorgabe der nächsten Schritte und die notwendige Information um das innere Chaos der Betroffenen etwas $\mathrm{zu}$ reduzieren. Information hilft gegen die Gefahr von Kontrollverlust.

Wenn möglich Betroffenen kleine Aufgaben zu geben, denn Handeln hilft auch gegen Hilflosigkeit. Durch 
gezielte Interventionen rationales Denken zu fördern ist eine Intervention gegen die überschwemmenden Gefühle.

\section{Interventionsschritte in Notfällen}

1. Beruhigen: Sicherheit vermitteln, Selbst ruhig bleiben und Erfahrung im Umgang mit Notfällen vermitteln;

2. Orientieren: den Betroffenen im Hier und Jetzt orientieren, über die nächsten Schritte und die aktuelle Lage informieren;

3. Ressourcen aktivieren: die persönlichen und sozialen Ressourcen zugänglich machen und fördern;

4. Wahrnehmung: Intensität reduzieren, ungewöhniche Wahrnehmungen erklären;

5. Zeiterleben: das Ereignis strukturieren und in einen zeitlichen Kontext stellen;

6. Gedankliche Verarbeitung: die kognitive Verarbeiung des Ereignisses und der eigenen Reaktionen fördern;

7. Gefühle: emotionale Entlastung ermöglichen, auf belastende Gefühle eingehen;

8. Kontrollierbarkeit: subjektive Kontrolle wiederherstellen und fördern;

9. Selbstbild: die Reaktionen normalisieren, Handlungsfähigkeit und Bewältigungsmöglichkeiten fördern;

10.Die nächsten Schritte: die kommenden Ereignisse und die weiteren Schritte besprechen, einen Plan für die nächsten Tage machen.

\section{Gefahren für psychosoziale NotfallhelferInnen}

Psychosoziale NotfallhelferInnen gehen in extreme Situationen und sind ebenfalls diesen Einflüssen ausgesetzt. Es ist sehr wichtig in der Einsatzleitung und im internen Stab folgende Belastungsfaktoren im Fokus der Aufmerksamkeit zu haben:

\section{Chaos}

De KollegInnen haben meist Zeitdruck, haben oft fehlende oder widersprüchliche Informationen und schnell wechselnde Einsatzlagen

\section{Gefahr}

E gibt immer wieder Situationen mit Gefährdungsotential von Gesundheit, aber auch die Gefahr des Scheiterns schwingt im Einsatz immer wieder mit

\section{Mangel}

Wenn die Ausrüstung nicht ausreichend ist, die Unterstützung im Einsatz mangelhaft und die Zuständigkeiten nicht geklärt sind das zusätzliche Belastungsfaktoren, auch wenn die Ausbildung nicht ausreichend ist.

\section{Gefühlsdruck}

Die Konfrontation mit verletzten, toten, trauernden, geschockten, hilflosen Menschen, aufwühlende Erlebnisse erzeugt oft einen enormen Gefühlsdruck

\section{Ungewissheit}

Ungewissheit bezüglich der Dauer des Einsatzes, die Art der weiteren Belastungen und dem Ausgang der Interventionen

Sicherheit und Planung sind wesentliche Parameter um oben genannte Belastungsfaktoren etwas reduzieren zu können.

Es braucht für die KollegInnen klare Ansprechpersonen, das heißt eine/n EinsatzleiterIn der eigenen Organisation vor Ort. Der Informationsfluss muss gewährleistet sein. Es ist darauf zu achten, dass vom/ von der EinsatzleiterIn vor Ort nur gesicherte Informationen weitergegeben werden.

Ein weiterer wesentlicher Punkt ist, dass die Einsatzdauer bekannt sein muss und Ablöse muss organsiert werden.

Nach dem Einsatz muss es das Angebot einer Einsatznachbesprechung, als klaren Abschluss des Einsatzes geben.

\section{Acknowledgements: None.}

Conflict of interest : None to declare.

\section{Literatur}

Sie können eine Liste der Literatur vom Autor erhalten.

Correspondence:

Monika Czamler, MD

Geschäftsfeldleitung der Krisenhilfe OÖ

Scharitzerstraße 6 - 8 /4. Stock, 4020 Linz, Austria

E-mail:monika.czamler@krisenhilfeooe.at 